\title{
Optimal High-Order Methods for Solving Nonlinear Equations
}

\author{
S. Artidiello, ${ }^{1}$ A. Cordero, ${ }^{2}$ Juan R. Torregrosa, ${ }^{2}$ and M. P. Vassileva $^{1}$ \\ ${ }^{1}$ Instituto Tecnológico de Santo Domingo (INTEC), Avenida de Los Próceres, Galá, 10601 Santo Domingo, Dominican Republic \\ ${ }^{2}$ Instituto de Matemática Multidisciplinar, Universitat Politècnica de València, Camino de Vera s/n, 40022 Valencia, Spain
}

Correspondence should be addressed to M. P. Vassileva; maria.vassilev@gmail.com

Received 4 February 2014; Accepted 7 April 2014; Published 5 May 2014

Academic Editor: Ioannis K. Argyros

Copyright (C) 2014 S. Artidiello et al. This is an open access article distributed under the Creative Commons Attribution License, which permits unrestricted use, distribution, and reproduction in any medium, provided the original work is properly cited.

\begin{abstract}
A class of optimal iterative methods for solving nonlinear equations is extended up to sixteenth-order of convergence. We design them by using the weight function technique, with functions of three variables. Some numerical tests are made in order to confirm the theoretical results and to compare the new methods with other known ones.
\end{abstract}

\section{Introduction}

The rapid advances in the development of digital computer have established the need to design new methods with higher computational efficiency for solving problems of practical relevance for applied mathematics, engineering, biology, and so forth. A variety of problems in different fields of science and technology require finding the solution of a nonlinear equation. Iterative methods for approximating solutions are the most used technique. The interest in the multipoint iterative methods has been renewed in the first decade of the 21st century as they are of great practical importance because they exceed the theoretical limits of the methods of a point on the order of convergence and computational efficiency.

Throughout this paper we consider multipoint iterative methods to find a simple root $\xi$ of a nonlinear equation $f(x)=0$, where $f: I \subset \mathbb{R} \rightarrow \mathbb{R}$, restricted to real functions with a unique solution inside an open interval $I$. Many modified schemes of Newton's method, probably the most widely used iterative method, have been proposed to improve the local order of convergence and the efficiency index over the last years. The efficiency index, introduced by Ostrowski in [1] as $I=p^{1 / d}$, where $p$ is the order of convergence and $d$ the number of functional evaluations per step, establishes the effectiveness of the iterative method. In this sense, Kung and Traub conjectured in [2] that a multipoint iterative scheme without memory, requiring $d+1$ functional evaluations per iteration, has order of convergence at most $2^{d}$. The schemes which achieve this bound are called optimal methods.

A common way to increase the convergence order in multipoint methods is to use weight functions that are applied to construct families of iterative methods for nonlinear equations. See, for example, the text by Petković et al. [3] and the references therein. The main goal and motivation in the construction of new methods is to attain as high as possible computational efficiency. Optimal methods of order four were discussed, for example, in [4, 5]. Many optimal methods of order eight have been suggested and compared in the literature; see, for instance, the recent results obtained by Kim in [6], Khan et al. in [7], Džunić and Petković in [8], and Soleymani et al. in [9]. Recently, by using weight function method some sixteenth-order iterative schemes have been also published as $[10,11]$.

The outline of the paper is as follows. In Section 2 the families of optimal sixteenth-order methods are constructed and the convergence analysis is discussed. In Section 3 numerical experiments are performed and the proposed methods of order sixteen are compared with the mentioned sixteenth-order schemes on academic test functions. Finally, in Section 4, the problem of preliminary orbit determination of artificial satellites is studied by using the classical fixed point method and numerical experiments on the modified Gaussian preliminary orbit determination are performed and the proposed methods are compared with recent optimal known schemes. 


\section{Description of the Family of Optimal Multipoint Methods}

Our starting point is Traub's scheme (see [12], also known as Potra-Pták's method) whose iterative expression is

$$
x_{k+1}=y_{k}-\frac{f\left(y_{k}\right)}{f^{\prime}\left(x_{k}\right)}=x_{k}-\frac{f\left(x_{k}\right)+f\left(y_{k}\right)}{f^{\prime}\left(x_{k}\right)} \text {, }
$$

where $y_{k}$ is Newton's step. This method has order three but it requires three functional evaluations, so it is not optimal according to Kung-Traub conjecture and our purpose is to design optimal methods. [13])

So, we begin the process from the iterative scheme (see

$$
\begin{gathered}
y_{k}=x_{k}-\beta \frac{f\left(x_{k}\right)}{f^{\prime}\left(x_{k}\right)}, \\
x_{k+1}=y_{k}-H\left(u\left(x_{k}\right)\right) \frac{f\left(y_{k}\right)}{f^{\prime}\left(x_{k}\right)},
\end{gathered}
$$

where $\beta$ is a real parameter and $H(u)$ is a real function with $u=f(y) / f(x)$.

The method defined by (2) has order four if $\beta=1$ and a function $H$ is chosen so that the conditions $H(0)=1$, $H^{\prime}(0)=2$, and $\left|H^{\prime \prime}(0)\right|<\infty$ are fulfilled. Some known iterative schemes are obtained as particular cases of this family. Choosing $H(u)=1 /(1-u)^{2}$, we obtain the fourthorder method described by Kung and Traub in [2]. King's family [14] of fourth-order methods is obtained when we choose $H(u)=(1+\beta u) /(1+(\beta-2) u)$. Also, if we take $H(u)=\left(1+2 u+\beta u^{2}\right) /\left(1+(\beta-5) u^{2}\right)$, we obtain the family of fourth-order methods defined by Zhao et al. in [15].

Recently, taking (2) with $\beta=1$ as the first two steps and adding a new step, Džunić et al. in [16] designed the following three-step method:

$$
\begin{gathered}
z_{k}=y_{k}-H\left(u\left(x_{k}\right)\right) \frac{f\left(y_{k}\right)}{f^{\prime}\left(x_{k}\right)}, \\
x_{k+1}=z_{k}-G\left(u\left(x_{k}\right), v\left(x_{k}\right)\right) \frac{f\left(z_{k}\right)}{f^{\prime}\left(x_{k}\right)},
\end{gathered}
$$

where $y_{k}$ is Newton's step and $G(u, v)$ is a function of two variables: $u=f(y) / f(x)$ and $v=f(z) / f(y)$.

They proved in [16] that the method defined by (3) has optimal eighth-order of convergence, if sufficiently differentiable functions $H$ and $G$ are chosen so that the conditions

$$
\begin{aligned}
& H(0)=1, \quad H^{\prime}(0)=2, \quad G(0,0)=1, \\
& G_{u}(0,0)=2, \quad G_{v}(0,0)=1, \\
& G_{u u}(0,0)=2+H^{\prime \prime}(0), \quad G_{u v}(0,0)=4,
\end{aligned}
$$

and $G_{u u u}(0,0)=-24+6 H^{\prime \prime}(0)+H^{\prime \prime \prime}(0)$ are satisfied. The iterative method resulting from introducing these conditions and the simplest form for $H$ and $G$, obtained by using the
Taylor polynomial of the functions: $H(u)=1+2 u$ and $G(u, v)=1+2 u+v+u^{2}+4 u v-4 u^{3}$, is denoted by $M 8$.

Now, we wonder if it is possible to find a sixteenth-order iterative method by adding a new step with the same settings accompanied with a weight function $T$ that depends on three variables $u$, $v$, and $w=f(s) / f(z)$, where $s$ is the last step of the eighth-order method (3). The iterative expression of the new scheme is

$$
\begin{gathered}
s_{k}=z_{k}-G\left(u\left(x_{k}\right), v\left(x_{k}\right)\right) \frac{f\left(z_{k}\right)}{f^{\prime}\left(x_{k}\right)}, \\
x_{k+1}=s_{k}-T\left(u\left(x_{k}\right), v\left(x_{k}\right), w\left(x_{k}\right)\right) \frac{f\left(s_{k}\right)}{f^{\prime}\left(x_{k}\right)},
\end{gathered}
$$

where $y_{k}$ and $z_{k}$ are the same steps as in method (3). The following result can be proved that establishes the sixteenthorder of family (5).

Theorem 1. Let $\xi \in I$ be a simple zero of a sufficiently differentiable function $f: I \subset \mathbb{R} \rightarrow \mathbb{R}$ in an open interval $I$ and $x_{0}$ an initial guest close to $\xi$. The method defined by (5) has optimal sixteenth-order convergence if sufficiently differentiable functions $H, G$, and $T$ are chosen so that the conditions on method (3) (proved in [16]) and the following requirements are satisfied:

$$
\begin{array}{cc}
H^{\prime \prime}(0)=0, & H^{(3)}=24, \\
H^{(4)}(0)=-72, & G_{u u}(0,0)=2, \\
G_{u u u}(0,0)=0, & G_{u u u v}(0,0)=24, \\
G_{u u v v}(0,0)=-16, & G_{u u v}(0,0)=6, \\
G_{u u u u}(0,0)=0, & T(0,0,0)=1, \\
T_{u}(0,0,0)=2, \quad T_{v}(0,0,0)=1, \\
T_{w}(0,0,0)=1, \quad T_{u u}(0,0,0)=2, \\
T_{u v}(0,0,0)=4, \quad T_{v v}(0,0,0)=G_{v v}(0,0), \\
T_{v w}(0,0,0)=2, \quad T_{u u v}(0,0,0)=8, \\
T_{u u u}(0,0,0)=0, \quad T_{u v v}(0,0,0)=4+G_{u v v}(0,0), \\
T_{u v v}(0,0,0)=8, \quad T_{u w}(0,0,0)=2, \\
T_{u u w}(0,0,0)=2,
\end{array}
$$

$G_{u v v}(0,0)=8-(1 / 3)\left(G_{u v v v}(0,0)+6 G_{v v}(0,0)\right)$, and $T_{v v v}(0$, $0,0)=-6+3 G_{v v}(0,0)+G_{v v v}(0,0)$. The error equation of the method is

$$
\begin{aligned}
e_{k+1}= & -\frac{1}{48} c_{2}\left(5 c_{2}^{2}-c_{3}\right)\left(\alpha_{1}-2 c_{2} c_{4}\right) \\
& \times\left(\alpha_{2}+6 c_{3} c_{5}\right) e_{k}^{16}+\mathcal{O}\left(e_{k}^{17}\right),
\end{aligned}
$$


where

$$
\begin{aligned}
\alpha_{1} & =5 M_{1} c_{2}^{4}+2 M_{2} c_{2}^{2} c_{3}+M_{3} c_{3}^{2}-2 c_{2} c_{4}, \\
\alpha_{2}= & 25 N_{1} c_{2}^{8}-20 N_{2} c_{2}^{6} c_{3} N_{3} c_{3}^{4}+60 N_{4} c_{2}^{5} c_{4} \\
& +24 N_{5} c_{2}^{3} c_{3} c_{4}+12 N_{6} c_{2} c_{3}^{2} c_{4} \\
& +6 c_{2}^{4}\left(N_{7} c_{3}^{2}+20 c_{5}\right)-4 c_{2}^{2}\left(N_{8} c_{3}^{3}+3 N_{9}\right) c_{4}^{2} \\
& +6 c_{3} c_{5}
\end{aligned}
$$

$c_{k}=(1 / k !)\left(f^{(k)}(\xi) / f^{\prime}(\xi)\right), k=2,3, \ldots, e_{k}=x_{k}-\xi$, and $M_{i}, N_{i}, i=1,2, \ldots, 8$ depend on the partial derivatives of order one, two, and three of the weight functions $G$ and $T$ at zero.

Proof. The proof is based on Taylor's expansion of the elements appearing in the iterative expression (5). We only show the necessary elements of the expressions in order to determine the conditions needed to attain the order of convergence. The Taylor expansion of the weight functions used is developed around zero but, for the sake of simplicity, we will omit the zero in the Taylor expansion of $H, G$, and $T$.

By using Taylor's expansion about $\xi$, we have $f\left(x_{k}\right)=$ $f^{\prime}(\xi) \sum_{j=1}^{16} c_{j} e_{k}^{j}+\mathcal{O}\left(e_{k}^{17}\right)$, where $c_{1}=1$ and $f^{\prime}\left(x_{k}\right)=f^{\prime}(\xi)(1+$ $\left.\sum_{j=2}^{15} j c_{j} e_{k}^{j-1}\right)+\mathcal{O}\left(e_{k}^{16}\right)$. By substituting the expression in the first step of (5), we obtain $y_{k}-\xi=\sum_{j=2}^{16} A_{j} e_{k}^{j}+\mathcal{O}\left(e_{k}^{16}\right)$, where $A_{2}=c_{2}, A_{3}=2\left(c_{3}-2 c_{2}^{2}\right)$, and $A_{4}=4 c_{2}^{3}-7 c_{2} c_{3}+$ $3 c_{4}$. Using again Taylor's expansion, we obtain $f\left(y_{k}\right)$ and we calculated $u\left(x_{k}\right)=f\left(x_{k}\right) / f^{\prime}\left(x_{k}\right)$ and $H\left(u\left(x_{k}\right)\right) \approx 1+$ $2 u\left(x_{k}\right)+(1 / 2) H^{\prime \prime}(0) u\left(x_{k}\right)^{2}+(1 / 6) H^{(3)}(0) u\left(x_{k}\right)^{3}+(1 /$ 24) $H^{(4)}(0) u\left(x_{k}\right)^{4}$, where we demand conditions $H(0)=1$ and $H^{\prime}(0)=2$. This allows us to obtain the error equation (fourth-order) for the second step $z_{k}: z_{k}-\xi=\sum_{j=4}^{16} B_{j} e_{k}^{j}+$ $\mathcal{O}\left(e_{k}^{16}\right)$, where $B_{4}=\left(5-H^{\prime \prime}(0) / 2\right) c_{2}^{3}-c_{2} c_{3}$. We use again Taylor's expansion about $\xi$ for obtaining $f\left(z_{k}\right)$, calculate $v\left(x_{k}\right), G\left(u\left(x_{k}\right), v\left(x_{k}\right)\right)$, introduce the known conditions ([16]): $G(0,0)=1, G_{u}(0,0)=2, G_{v}(0,0)=1, G_{u u}(0,0)=2+$ $H^{\prime \prime}(0), G_{u v}(0,0)=4$ and $G_{u u u}(0,0)=-24+6 H^{\prime \prime}(0)+H^{(3)}(0)$, and obtain Taylor's series of $s_{k}$ :

$$
s_{k}-\xi=\sum_{j=8}^{16} S_{j} e_{k}^{j}+\mathcal{O}\left(e_{\mathrm{k}}^{17}\right)
$$

where

$$
\begin{aligned}
S_{8}=-\frac{1}{48} s_{8,1}\left(s_{8,2} c_{2}^{4}+s_{8,2} c_{2}^{2} c_{3}+s_{8,4} c_{3}^{2}+24 c_{2} c_{4}\right), \\
s_{8,1}=\alpha c_{2}^{3}+2 c_{2} c_{3}, \\
s_{8,2}=1080-G_{u u u u}(0,0)-300 G_{v v}(0,0)+6 \alpha G_{u u v}(0,0) \\
\times\left[-108+3 G_{v v}(0,0)\left(20-H^{\prime \prime}(0)\right) H^{\prime \prime}(0)\right] \\
+8 H^{(3)}(0)+H^{(4)}(0),
\end{aligned}
$$

$$
\begin{gathered}
s_{8,3}=-12\left(38-G_{u u v}(0,0)+\alpha G_{v v}(0,0)-H^{\prime \prime}(0)\right), \\
s_{8,4}=12\left(2-G_{v v}(0,0)\right),
\end{gathered}
$$

and $\alpha=H^{\prime \prime}(0)-10$. So, using again Taylor's expansion about $\xi$, we obtain $f\left(s_{k}\right)$ and use it to get Taylor's expression of $w\left(x_{k}\right)$ and $T\left(u\left(x_{k}\right), v\left(x_{k}\right), w\left(x_{k}\right)\right)$. Finally, we obtain the error equation of the proposed iterative scheme (5): $e_{k+1}=\sum_{j=8}^{16} N_{j} e_{k}^{j}+$ $\mathcal{O}\left(e_{k}^{17}\right)$, where $N_{8}=[1-T(0,0,0)] S_{8}$. If $T(0,0,0)=1$, then $N_{8}=0$ and $e_{k+1}=\sum_{j=9}^{16} N_{1, j} e_{k}^{j}+\mathcal{O}\left(e_{k}^{17}\right)$, where $N_{1,9}=$ $\left[2-T_{u}(0,0,0)\right] S_{8}$. Taking $T_{u}(0,0,0)=2$, we obtain $e_{k+1}=$ $\sum_{j=10}^{16} N_{2, j} e_{k}^{j}+\mathcal{O}\left(e_{k}^{17}\right)$, where $N_{2,10}=(1 / 2)\left[\left(12+\alpha T_{v}(0,0,0)-\right.\right.$ $\left.\left.T_{u u}(0,0,0)\right) c_{2}^{2}+2\left(T_{u u}(0,0,0)-1\right) c_{3}\right] S_{8}$. If we make $T_{v}(0,0,0)=$ 1 and $T_{u u}(0,0,0)=2+H^{\prime \prime}(0)$, we ensure order of convergence is at least eleven. The error equation in this case takes the following form: $e_{k+1}=\sum_{j=11}^{16} N_{3, j} e_{k}^{j}+\mathcal{O}\left(e_{k}^{17}\right)$ and $N_{3,11}=-(1 / 6) \mathcal{c}_{2}\left[\left(96+H^{(3)}(0)-T_{u u u}(0,0,0)+3 H^{\prime \prime}(0)\left(T_{u v}(0\right.\right.\right.$, $\left.\left.0,0)-2)-30 T_{u v}(0,0,0)\right) c_{2}^{2}+6\left(T_{u v}(0,0,0)-4\right) c_{3}\right] S_{8}$. Taking $T_{u v}(0,0,0)=4$ and $H^{(3)}(0)=24-6 H^{\prime \prime}(0)+T_{u u u}(0,0,0)$, we obtain the new expression

$$
e_{k+1}=\sum_{j=12}^{16} N_{4, j} e_{k}^{j}+\mathcal{O}\left(e_{k}^{17}\right)
$$

where

$$
\begin{gathered}
N_{4,12}=n\left[n_{12,2} c_{2}^{4}+n_{12,3} c_{2}^{2} c_{3}\right. \\
\left.\quad+n_{12,4} c_{3}^{2}-\left(T_{w}(0,0,0)-1\right) c_{2} c_{4}\right], \\
n=\frac{1}{1152}\left(\alpha c_{2}^{3}+2 c_{3}\right)\left[n_{12,1}-24 c_{2} c_{4}\right],
\end{gathered}
$$

$n_{12,2}$

$$
\begin{aligned}
& =1392+H^{(4)}(0)-60 T_{u u v}(0,0,0)+8 T_{u u u}(0,0,0) \\
& +30 T_{v v}(0,0,0)-\left(1272-60 G_{u u v}(0,0)\right. \\
& -G_{\text {uии }}(0,0)-300 G_{v v}(0,0) \\
& \left.-H^{(4)}(0)+8 T_{u u u}(0,0,0)\right) T_{w}(0,0,0) \\
& -6 H^{\prime \prime}(0)\left[28-T_{u u v}(0,0,0)-10 T_{v v}(0,0,0)\right. \\
& \left.-\left(26-G_{u u v}(0,0)-10 G_{v v}(0,0)\right) T_{w}(0,0,0)\right] \\
& -3 H^{\prime \prime}(0)^{2}\left(T_{v v}(0,0,0)-G_{v v}(0,0) T_{w}(0,0,0)\right) \text {, } \\
& n_{12,3}=-12 \\
& \times\left[40-T_{u u v}(0,0,0)-10 T_{v v}(0,0,0)\right. \\
& \left.-\left(38-G_{u u v}(0,0)-10 G_{v v}(0,0)\right) T_{w}(0,0,0)\right] \\
& +12 H^{\prime \prime}(0)\left(1-T_{v v}(0,0,0)-T_{w}(0,0,0)\right. \\
& \left.+G_{v v}(0,0) T_{w}(0,0,0)\right), \\
& n_{12,4}=12\left[2-T_{v v}(0,0,0)+\left(G_{v v}(0,0)-2\right) T_{w}(0,0,0)\right] \text {. }
\end{aligned}
$$

If $n_{12,2}=n_{12,3}=n_{12,4}=0$ and $T_{w}(0,0,0)-1=0$, the order of convergence is at least thirteen. The solution of these 
four equations determine that $T_{\mathrm{u} u v}(0,0,0)=2+G_{u u v}(0,0)$, $T_{v v}(0,0,0)=G_{v v}(0,0)$, and $T_{w}(0,0,0)=1$ and the error equation is

$$
e_{k+1}=\sum_{j=13}^{16} N_{5, j} e_{k}^{j}+\mathcal{O}\left(e_{k}^{17}\right) \text {, }
$$

where

$$
\begin{aligned}
& N_{5,13}=n c_{2}\left[n_{13,1} c_{2}^{4}+n_{13,2} c_{2}^{2} c_{3}+n_{13,3} c_{3}^{2}\right. \\
& \left.-24\left(T_{u w}(0,0,0)-2\right) c_{2} c_{4}\right], \\
& n_{13,1}=3254-2 \alpha G_{u u u v}(0,0)-384 H^{\prime \prime}(0) \\
& +2 H^{(4)}(0)-6 \alpha G_{u u v}(0,0)\left(T_{u w}(0,0,0)-2\right) \\
& +16 T_{\text {uиu }}(0,0,0) \\
& +3\left(100-20 H^{\prime \prime}(0)+H^{\prime \prime}(0)^{2}\right) G_{u v v}(0,0) \\
& -6\left(100-20 H^{\prime \prime}(0)+H^{\prime \prime}(0)^{3}\right) G_{v v}(0,0) \\
& -\left(1278+300 G_{v v}(0,0)+156 H^{\prime \prime}(0)\right. \\
& \left.-60 G_{v v}(0,0) H^{\prime \prime}(0)-8 T_{\text {uนu }}(0,0,0)\right) \\
& \times T_{u w}(0,0,0) \\
& +\left(36 G_{v v}(0,0) H^{\prime \prime}(0)-H^{(4)}(0)\right) T_{u w}(0,0,0), \\
& n_{13,2}=4\left[G_{u u u v}(0,0)\right. \\
& +3\left(G_{u u v}(0,0)+\alpha G_{v v}(0,0)\right)\left(T_{u w}(0,0,0)-2\right) \\
& +3\left(108-\alpha G_{u v v}(0,0)-4 H^{\prime \prime \prime}(0)\right. \\
& \left.\left.-\left(38-H^{\prime \prime}(0)\right) T_{u v}(0,0,0)\right)\right], \\
& n_{13,3}=12\left[8+G_{u v v}(0,0)+G_{v v}(0,0)\right. \\
& \left.\times\left(T_{u w}(0,0,0)-2\right)-2 T_{u w}(0,0,0)\right] .
\end{aligned}
$$

For obtaining order of convergence of at least fourteen it is necessary that $n_{13,1}=n_{13,2}=n_{13,3}=0$ and $T_{u w}(0,0,0)-2=0$. This gives us the conditions: $T_{u v}(0,0,0)=2, G_{u v v}(0,0)=-4$, and $G_{u u u v}(0,0)=-6\left(H^{\prime \prime}(0)-4\right)$ and the error equation is

$$
e_{k+1}=\sum_{j=14}^{16} N_{6, j} e_{k}^{j}+\mathcal{O}\left(e_{k}^{17}\right) \text {, }
$$

where

$$
\begin{aligned}
N_{6,13}=n[ & n_{14,1} c_{2}^{6}-2 n_{14,2} c_{2}^{4} c_{3}-12 n_{14,3} c_{2}^{2} c_{3}^{2} \\
& +8 n_{14,4} c_{3}^{3}-24 n_{14,5} c_{2}^{3} c_{4} \\
& \left.-48\left(T_{v w}(0,0,0)-2\right) c_{2} c_{3} c_{4}\right] .
\end{aligned}
$$

$$
\text { If } T_{v w}(0,0,0)=2 \text {, }
$$$$
n_{14,1}=2976-2400 G_{v v}(0,0)
$$$$
-100 G_{v w}(0,0)-3 \alpha^{2} G_{u u v v}(0,0)
$$$$
-\left(2112-1080 G_{v v}(0,0)-300 G_{v v v}(0,0)\right) H^{\prime \prime}(0)
$$$$
+\left(216-144 G_{v v}(0,0)-30 G_{v v v}(0,0)\right) H^{\prime \prime}(0)^{2}
$$$$
+\left(6 G_{v v}(0,0)+G_{v v v}(0,0)\right) H^{\prime \prime}(0)^{3}+8 H^{(4)}(0)
$$$$
-H^{\prime \prime}(0) H^{(4)}(0)-6 \alpha G_{u u v}(0,0)
$$$$
\times\left(8-2 H^{\prime \prime}(0)+T_{\text {uuw }}(0,0,0)\right)
$$$$
+\left(1272-300 G_{v v}(0,0)-156 H^{\prime \prime}(0)\right.
$$$$
+60 G_{v v}(0,0) H^{\prime \prime}(0)
$$$$
\left.-3 G_{v v}(0,0) H^{\prime \prime}(0)^{2}+H^{(4)}(0)\right) T_{u u w}(0,0,0)
$$$$
-8\left(2+H^{\prime \prime}(0)-T_{\text {uиw }}(0,0,0)\right) T_{\text {uии }}(0,0,0)
$$$$
+\left(1000-300 H^{\prime \prime}(0)+3 H^{\prime \prime}(0)^{2}-H^{\prime \prime}(0)^{3}\right)
$$$$
\times T_{v v}(0,0,0),
$$$$
n_{14,2}=10176-780 G_{v v}(0,0)
$$$$
-300 G_{v v v}(0,0)-6 \alpha G_{u u v v}(0,0)
$$$$
-\left(144-228 G_{v v}(0,0)+60 G_{v v v}(0,0)\right) H^{\prime \prime}(0)
$$$$
+\left(12-15 G_{v v}(0,0)-3 G_{v v v}(0,0)\right) H^{\prime \prime}(0)^{2}
$$$$
+H^{(4)}(0)-6 G_{u u v}(0,0)
$$$$
\times\left(18-3 H^{\prime \prime}(0)+T_{\text {uนw }}(0,0,0)\right)
$$$$
+6\left(38+\alpha G_{v v}(0,0)-H^{\prime \prime}(0)\right) T_{u u w}(0,0,0)
$$$$
+3\left(100-20 H^{\prime \prime}(0)+H^{\prime \prime}(0)^{2}\right) T_{v v v}(0,0,0),
$$$$
n_{14,3}=-52+2 G_{u u v}(0,0)+G_{u u v v}(0,0)
$$$$
+6 H^{\prime \prime}(0)+4 G_{v v}(0,0)\left(7-H^{\prime \prime}(0)\right)
$$$$
-\alpha G_{v v v}(0,0)+\alpha T_{v v v}(0,0,0)
$$$$
+T_{u u w}(0,0,0)\left(G_{v v}(0,0)-2\right) \text {, }
$$$$
n_{14,4}=6-3 G_{v v}(0,0)-G_{v v v}(0,0)+T_{v v v}(0,0,0) \text {, }
$$

$$
n_{14,5}=2+H^{\prime \prime}(0)-T_{u u w}(0,0,0) \text {. }
$$

Now, if we demand $n_{14,1}=n_{14,2}=n_{14,3}=n_{14,4}=n_{14,5}=0$, the order of convergence is at least fifteen, and the necessary 
conditions are $T_{u u w}(0,0,0)=2+H^{\prime \prime}(0), G_{v v v}(0,0,0)=$ $6-3 G_{v v}(0,0)+T_{v v v}(0,0,0), G_{u u v v}(0,0)=-2\left(2+G_{u u v}(0,0)-\right.$ $\left.H^{\prime \prime}(0)\right), H^{(4)}(0)=12\left(H^{\prime \prime}(0)-6\right)$. Taking into account these conditions, the error equation is

$$
e_{k+1}=N_{7,15} e_{k}^{15}+N_{7,16} e_{k}^{16}+\mathcal{O}\left(e_{k}^{17}\right)
$$

where $N_{7,15}=-(1 / 6) n c_{2}\left[\left(n_{15,2} c_{2}^{6}-n_{15,3} c_{2}^{4} c_{3}+n_{15,4} c_{2}^{2} c_{3}^{2}+\right.\right.$ $\left.24 n_{15,5} c_{3}^{3}-n_{15,6} c_{2}^{3} c_{4}-144\left(T_{u v w}(0,0,0)-8\right) c_{2} c_{3} c_{4}\right]$. By taking $T_{u v w}(0,0,0)=8$ and simplifying the error equation, we obtain

$$
\begin{aligned}
e_{k+1}= & -\frac{1}{6912} c_{2}^{2}\left(\alpha c_{2}^{2}+2 c_{3}\right) \\
& \times\left[n_{15,7}-24 c_{2} c_{4}\right]\left[n_{15,8}-24 T_{\text {uиu }}(0,0,0) c_{2}^{3} c_{4}\right] \\
& \times e_{k}^{15}+N_{8,16} e_{k}^{16}+\mathcal{O}\left(e_{k}^{17}\right)
\end{aligned}
$$

and $T_{\text {uиu }}(0,0,0)=0$; we have

$$
\begin{aligned}
e_{k+1}= & -\frac{1}{768} c_{2}^{2}\left(\alpha c_{2}^{2}+2 c_{3}\right)^{2} \\
& \times\left[n_{15,9}-8 c_{2} c_{4}\right]\left[n_{15,10} c_{2}^{4}+4 n_{15,11} c_{2}^{2} c_{3}+4 n_{15,12} c_{3}^{2}\right] \\
& +N_{8,16} e_{k}^{16}+\mathcal{O}\left(e_{k}^{17}\right) .
\end{aligned}
$$

By solving the system

$$
\begin{aligned}
n_{15,10}= & 12 \alpha G_{u u v}(0,0)+\alpha^{2} G_{u v v v}(0,0) \\
& +6\left[\alpha^{2} G_{v v}(0,0)-4\left(180-3 H^{\prime \prime}(0)+H^{\prime \prime}(0)^{2}\right)\right. \\
= & , \\
n_{15,11}= & 396-6 G_{u u v}(0,0) \\
& -10 G_{u v v v}(0,0)-60 G_{v v}(0,0) \\
& \quad\left(30-G_{u v v v}(0,0)-6 G_{v v}(0,0)\right) H^{\prime \prime}(0)=0, \\
n_{15,12}= & G_{u v v}(0,0)+6\left(G_{v v}(0,0)-6\right)=0,
\end{aligned}
$$

we obtain $G_{u v v v}(0,0)=-6\left(-6+G_{v v}(0,0)\right), H^{\prime \prime}(0,0)=0$, and $G_{u u v}(0,0)=6$. Finally, the error equation is

$$
\begin{aligned}
e_{k+1}= & \frac{1}{48} c_{2}\left(5 c_{2}^{2}-c_{3}\right)\left[\alpha_{1}-2 c_{2} C_{3}\right]\left[\alpha_{2}+6 C_{3} c_{5}\right] e_{k}^{16} \\
& +\mathcal{O}\left(e_{k}^{17}\right) .
\end{aligned}
$$

This finishes the proof.
A particular element of family (5), denoted by M16, is obtained by choosing the weight functions:

$$
\begin{gathered}
H(u)=1+2 u+4 u^{3}-3 u^{4} \\
G(u, v)=1+2 u+v+u^{2}+4 u v \\
+3 u^{2} v+4 u v^{2}+4 u^{3} v-4 u^{2} v^{2} \\
T(u, v, w)=1+2 u+v+w+u^{2}+4 u v \\
+2 u w+4 u^{2} v+u^{2} w+6 u v^{2} \\
+8 u v w-v^{3}+2 v w
\end{gathered}
$$

which we will use in the following sections.

\section{Numerical Tests for Sixteenth-Order Methods}

The proposed iterative scheme with order of convergence sixteen M16 is employed to estimate the simple solution of some particular nonlinear equations. It will be compared with some known methods existing in the literature. In particular, the iterative scheme of the sixteenth-order scheme designed by Thukral in $[10]$ is

$$
z_{k}=y_{k}-\frac{f\left[w_{k}, x_{k}\right]}{f\left[w_{k}, y_{k}\right]} \frac{f\left(y_{k}\right)}{f\left[x_{k}, y_{k}\right]},
$$

$$
a_{k}=z_{k}-\frac{1}{\left(1+2 u_{3} u_{4}^{2}\right)\left(1-u_{2}\right)}
$$

$$
\begin{gathered}
\times \frac{f\left(z_{k}\right)}{f\left[y_{k}, z_{k}\right]-f\left[x_{k}, y_{k}\right]+f\left[x_{k}, z_{k}\right]}, \\
x_{k+1}=a_{k}-T f\left(a_{k}\right) \frac{f\left[y_{k}, z_{k}\right]}{f\left[y_{k}, a_{k}\right] f\left[z_{k}, a_{k}\right]},
\end{gathered}
$$

where $y_{k}$ is Steffensen's step, $w_{k}=x_{k}+f\left(x_{k}\right), u_{1}=f\left(z_{k}\right) /$ $f\left(x_{k}\right), u_{2}=f\left(z_{k}\right) / f\left(w_{k}\right), u_{3}=f\left(y_{k}\right) / f\left(x_{k}\right), u_{4}=f\left(y_{k}\right) /$ $f\left(w_{k}\right), u_{5}=f\left(a_{k}\right) / f\left(x_{k}\right), u_{6}=f\left(a_{k}\right) / f\left(w_{k}\right)$, and $T=1+$ $u_{1} u_{2}-u_{1} u_{3} u_{4}^{2}+u_{5}+u_{6}+u_{1}^{2} u_{4}+u_{2}^{2} u_{3}+3 u_{1} u_{4}^{2}\left(\left(u_{3}^{2}-\right.\right.$ $\left.\left.u_{4}^{2}\right) / f\left[x_{k}, y_{k}\right]\right)$. We will denote this scheme by T16.

We will also use the sixteenth-order procedure designed by Sharma et al. in [11] that will be denoted by S16, whose iterative expression is

$$
\begin{gathered}
z_{k}=w_{k}-\frac{f\left(x_{k}\right)}{f\left(x_{k}\right)-2 f\left(w_{k}\right)} \frac{f\left(w_{k}\right)}{f^{\prime}\left(x_{k}\right)}, \\
t_{k}=x_{k}-\frac{f\left(x_{k}\right)(p+q+r)}{p f\left[z_{k}, x_{k}\right]+q f^{\prime}\left(x_{k}\right)+r f\left[w_{k}, x_{k}\right]}, \\
x_{k+1}=x_{k}-\frac{p_{1} f\left[z_{k}, w_{k}\right]+q_{1} f\left[w_{k}, x_{k}\right]+r f\left[t_{k}, w_{k}\right]}{p_{1} l+q_{1} m+r n} \\
\times f\left(x_{k}\right),
\end{gathered}
$$


where $w_{k}$ is Newton's step and

$$
\begin{gathered}
p=\left(x_{k}-w_{k}\right) f\left(x_{k}\right) f\left(w_{k}\right), \\
q=\left(w_{k}-z_{k}\right) f\left(z_{k}\right) f\left(w_{k}\right), \\
r=\left(z_{k}-x_{k}\right) f\left(z_{k}\right) f\left(x_{k}\right), \\
p_{1}=\left(x_{k}-t_{k}\right) f\left(x_{k}\right) f\left(t_{k}\right), \\
q_{1}=\left(t_{k}-z_{k}\right) f\left(t_{k}\right) f\left(z_{k}\right), \\
m=\frac{f\left(w_{k}\right) f\left[z_{k}, x_{k}\right]-f\left(z_{k}\right) f\left[w_{k}, x_{k}\right]}{w_{k}-z_{k}}, \\
m=\frac{f\left(w_{k}\right) f^{\prime}\left(x_{k}\right)-f\left(x_{k}\right) f\left[w_{k}, x_{k}\right]}{w_{k}-x_{k}}, \\
n=\frac{f\left(w_{k}\right) f\left[x_{k}, t_{k}\right]-f\left(t_{k}\right) f\left[w_{k}, x_{k}\right]}{w-t} .
\end{gathered}
$$

The numerical behavior will be analyzed by means of the test functions and the corresponding simple roots listed below:

(a) $f_{1}(x)=\log x^{2}+1+\exp (x) \sin (x), \xi=0$,

(b) $f_{2}(x)=1+\exp \left(x^{3}-x\right)-\cos \left(1-x^{2}\right)+x^{3}, \xi=-1$,

(c) $f_{3}(x)=(x-2)\left(x^{10}+x+1\right) \exp (-x-1), \xi=2$.

All the computations have been carried out by using variable precision arithmetics with 4000 digits of mantissa. The exact solution of the nonlinear equations is known, so the exact absolute error of the first three iterations of each procedure is listed in Table 1, joint with the computational order of convergence COC (see [17]), for different initial estimations $x_{0}$.

From results shown in Table 2, it can be deduced that the proposed scheme is, at least, as competitive as recently published methods of the same order of convergence, being better in some cases.

\section{Preliminary Orbit Determination}

A classical reference in preliminary orbit determination is $\mathrm{F}$. Gauss (1777-1855), who deduced the orbit of the minor planet Ceres, discovered in 1801 and afterwards lost. The so-called Gauss' method is based on the rate $y$ between the triangle and the ellipse sector defined by two position vectors from astronomical observations. This proportion is related to the geometry of the orbit and the observed position by

$$
y=1+X(l+x)
$$

where $l=\left(r_{1}+r_{2}\right) / 4 \sqrt{r_{1} r_{2}} \cos \left(\left(\nu_{2}-\nu_{1}\right) / 2\right)-(1 / 2), x=$ $\sin ^{2}\left(\left(E_{2}-E_{1}\right) / 4\right)$, and $X=\left(E_{2}-E_{1}-\sin \left(E_{2}-E_{1}\right)\right) / \sin ^{3}\left(\left(E_{2}-\right.\right.$ $\left.\left.E_{1}\right) / 2\right)$. The angles $E_{i}, v_{i}, i=1,2$, are the eccentric and true anomalies, respectively, associated with the observed positions $\overrightarrow{r_{1}}$ and $\overrightarrow{r_{2}}$ (let us denote by $r_{i}$ the modulus of vector $\left.\overrightarrow{r_{i}}, i=1,2\right)$.
Equation (27) is, actually, the composition of the First and Second Gauss Equation

$$
y^{2}=\frac{m}{l+x}, \quad y^{2}(y-1)=m X
$$

where $m=\mu \tau^{2} /\left[2 \sqrt{r_{1} r_{2}} \cos \left(\left(\nu_{2}-v_{1}\right) / 2\right)\right]^{3}, \mu$ is the gravitational parameter of the motion, and $\tau$ is a modified time variable.

The original iterative procedure used to solve the nonlinear Gauss equation (27) is the fixed point method (see, e.g., [18]) and is described in the following scheme.

(i) From the initial estimation $y_{0}=1, x_{0}=m / y_{0}^{2}-l$ is obtained (it is possible to calculate $m$ and $l$ from the observed positions $\overrightarrow{r_{1}}$ and $\overrightarrow{r_{2}}$ and the time $\tau$.

(ii) From $x_{0}$ and $\cos \left(\left(E_{2}-E_{1}\right) / 2\right)=1-2 x_{0}, \sin \left(\left(E_{2}-\right.\right.$ $\left.\left.E_{1}\right) / 2\right)=+\sqrt{4 x_{0}\left(1-x_{0}\right)}$, we calculate $E_{2}-E_{1}$. Then, we obtain $X_{0}=\left(E_{2}-E_{1}-\sin \left(E_{2}-E_{1}\right)\right) / \sin ^{3}\left(\left(E_{2}-\right.\right.$ $\left.E_{1}\right) / 2$ ).

(iii) By using the combined Gauss equation (27), a new iteration $y_{1}$ is calculated and the process starts again.

The iterative process follows as described above, getting new estimations of the ratio, until it does not vary within a given tolerance. Once the method has converged, the semimajor axis $a$ can be calculated by means of equation

$$
\begin{aligned}
y & =\frac{\sqrt{\mu p} \cdot \tau}{r_{2} r_{1} \sin \left(v_{2}-v_{1}\right)} \\
& =\frac{\sqrt{\mu} \cdot \tau}{2 \sqrt{a} \sqrt{r_{2} r_{1}} \sin \left(\left(E_{2}-E_{1}\right) / 2\right) \cos \left(\left(\nu_{2}-v_{1}\right) / 2\right)},
\end{aligned}
$$

from the last estimations of ratio and difference of eccentric anomalies, and the last phase is then initiated, to determine velocity and orbital elements.

Let us note that the original Gauss' scheme has a restriction when the angle formed by the two position vectors is greater than $\pi / 4$, since in this case the areas of the triangle and the ellipse sector are not similar.

Now, we are going to compare schemes M8 and M16 with other known ones of orders 8 and 16, respectively. In particular, we analyze the behavior of these methods to obtain the preliminary orbit of an artificial satellite.

All the iterative schemes introduced in the following are optimal in the sense of Kung-Traub's conjecture and have been designed with the weight function technique, so they are fully comparable with the new ones designed in this paper. Let us refer now to the procedure that Kim presents in [6]: a three-step eighth-order method, whose iterative expression is

$$
\begin{aligned}
z_{k}=y_{k}-\frac{1+u_{k}+2 / 3 u_{k}^{2}}{1-u_{k}-2 u_{k}^{2}} \frac{f\left(y_{k}\right)}{f^{\prime}\left(x_{k}\right)}, \\
x_{k+1}=z_{k}-\frac{1-2 u_{k}+v_{k}}{1-3 u_{k}-2 v_{k}} \\
\times \frac{f\left(z_{k}\right)}{f^{\prime}\left(x_{k}\right)+f\left[y_{k}, x_{k}, z_{k}\right]\left(z_{k}-x_{k}\right)},
\end{aligned}
$$


TABLE 1: Comparison of sixteenth-order schemes.

\begin{tabular}{|c|c|c|c|c|c|}
\hline Test functions & $x_{0}$ & & $\mathrm{~T} 16$ & S16 & M16 \\
\hline \multirow{4}{*}{$f_{1}(x)$} & \multirow{4}{*}{0.3} & $\left|x_{1}-\xi\right|$ & $2.079 e-5$ & $5.991 e-8$ & $5.987 e-5$ \\
\hline & & $\left|x_{2}-\xi\right|$ & $5.195 e-67$ & $3.791 e-112$ & $3.613 e-58$ \\
\hline & & $\left|x_{3}-\xi\right|$ & $1.202 e-1052$ & $1.74 e-1779$ & $1.125 e-909$ \\
\hline & & $\mathrm{COC}$ & 16.0 & 16.0 & 16.0 \\
\hline \multirow{4}{*}{$f_{1}(x)$} & \multirow{4}{*}{1} & $\left|x_{1}-\xi\right|$ & 0.05729 & $7.143 e-4$ & $1.549 e-2$ \\
\hline & & $\left|x_{2}-\xi\right|$ & $7.02 e-14$ & $6.056 e-47$ & $4.122 e-20$ \\
\hline & & $\left|x_{3}-\xi\right|$ & $1.483 e-202$ & $4.408 e-736$ & $9.269 e-301$ \\
\hline & & COC & 16.0 & 16.0 & 16.0 \\
\hline \multirow{4}{*}{$f_{2}(x)$} & \multirow{4}{*}{-2} & $\left|x_{1}-\xi\right|$ & 0.1511 & $6.887 e-3$ & $7.588 e-5$ \\
\hline & & $\left|x_{2}-\xi\right|$ & 4.801 & $2.374 e-38$ & $5.508 e-65$ \\
\hline & & $\left|x_{3}-\xi\right|$ & 0.8086 & $1.299 e-605$ & $3.5019 e-1023$ \\
\hline & & $\mathrm{COC}$ & - & 16.0 & 16.0 \\
\hline \multirow{4}{*}{$f_{2}(x)$} & \multirow{4}{*}{-3} & $\left|x_{1}-\xi\right|$ & 0.1002 & 0.3238 & $8.93 e-3$ \\
\hline & & $\left|x_{2}-\xi\right|$ & $1.153 e-5$ & $3.062 e-8$ & $8.602 e-32$ \\
\hline & & $\left|x_{3}-\xi\right|$ & $6.83 e-75$ & $7.209 e-224$ & $7.042 e-496$ \\
\hline & & COC & 16.0 & 16.0 & 15.99 \\
\hline \multirow{4}{*}{$f_{3}(x)$} & \multirow{4}{*}{2.1} & $\left|x_{1}-\xi\right|$ & $2.365 e-5$ & $4.299 e-11$ & $3.28 e-6$ \\
\hline & & $\left|x_{2}-\xi\right|$ & $2.087 e-51$ & $1.015 e-159$ & $4.371 e-74$ \\
\hline & & $\left|x_{3}-\xi\right|$ & $2.859 e-788$ & $9.445 e-2538$ & $4.319 e-1160$ \\
\hline & & $\mathrm{COC}$ & 16.0 & 16.0 & 16.0 \\
\hline
\end{tabular}

TABLE 2: Comparison of modified-Gauss schemes for Orbit I.

\begin{tabular}{lccccccc}
\hline & $\left|\left(x_{1}-x_{0}\right)\right|$ & $\left|F\left(x_{1}\right)\right|$ & $\left|\left(x_{2}-x_{1}\right)\right|$ & $\left|F\left(x_{2}\right)\right|$ & $\left|\left(x_{3}-x_{2}\right)\right|$ & $\left|F\left(x_{3}\right)\right|$ & ACOC \\
\hline FP & $0.6450 e-2$ & - & $0.8288 e-4$ & - & $0.1055 e-5$ & 1.002 \\
K8 & $0.6368 e-2$ & $0.2059 e-21$ & $0.2033 e-21$ & $0.6553 e-158$ & $0.647 e-158$ & $0.2164 e-1113$ & 7.001 \\
S8 & $0.6368 e-2$ & $0.1377 e-23$ & $0.1359 e-23$ & $0.5565 e-197$ & $0.5495 e-197$ & $0.3967 e-1584$ & 8.001 \\
M8 & $0.6368 e-2$ & $0.1382 e-23$ & $0.1365 e-23$ & $0.5791 e-197$ & $0.5718 e-197$ & $0.5488 e-1584$ & 8.000 \\
T16 & $0.6368 e-2$ & $0.2662 e-63$ & $0.2628 e-63$ & $0.1642 e-1045$ & NaN & NaN \\
S16 & $0.6368 e-2$ & $0.7454 e-48$ & $0.7361 e-48$ & $0.6647 e-783$ & $0.6563 e-783$ & 0.0 \\
M16 & $0.6368 e-2$ & $0.6998 e-47$ & $0.6910 e-47$ & $0.2286 e-766$ & $0.2258 e-766$ & 0.0 & 16.000 \\
\hline
\end{tabular}

where $y_{k}$ is Newton's step, $u_{k}=f\left(y_{k}\right) / f\left(x_{k}\right), v_{k}=f\left(z_{k}\right) /$ $f\left(x_{k}\right)$, and $f[\cdot, \cdot, \cdot]$ denotes the divided difference of order two. We will denote this scheme by K8.

We will also compare our new schemes with the method designed by Soleymani et al. in [9] (denoted by S8), initialized with Ostrowski's procedure,

$$
\begin{gathered}
z_{k}=y_{k}-\frac{f\left(x_{k}\right)}{f\left(x_{k}\right)-2 f\left(y_{k}\right)} \frac{f\left(y_{k}\right)}{f^{\prime}\left(x_{k}\right)} \\
x_{k+1}=z_{k} \\
-\frac{f\left(z_{k}\right)}{2 f\left[y_{k}, x_{k}\right]-f^{\prime}\left(x_{k}\right)+f\left[z_{k}, x_{k}, x_{k}\right]\left(z_{k}-y_{k}\right)} \\
\times\left(1+w_{k}+2 v_{k}-2 u_{k}^{3}+\frac{2}{5} \frac{f\left(z_{k}\right)}{f^{\prime}\left(x_{k}\right)}\right)
\end{gathered}
$$

where $y_{k}$ is Newton's step, $u_{k}=f\left(y_{k}\right) / f\left(x_{k}\right), v_{k}=f\left(z_{k}\right) /$ $f\left(x_{k}\right)$, and $w_{k}=f\left(z_{k}\right) / f\left(y_{k}\right)$.
The proposed iterative scheme M16 will be compared again with T16 and S16.

In the numerical test made, variable precision arithmetics has been used, with 4000 digits of mantissa in Matlab R2011b. Some reference orbits have been used in the test that can be found in [18]. As orbital elements of each one of the test orbits are known, the vector position in the instants $t_{1}$ and $t_{2}$ have been recalculated with 3998 exact digits. Then, our aim is to solve the unified Gauss equation from these positions, with the highest possible precision. In this term, the orbital elements can be calculated with the best accuracy.

(i) Test Orbit I has the position vectors

$\vec{r}_{1} \approx[2.46080928705339$, 2.04052290636432, 0.14381905768815$]$

$\vec{r}_{2} \approx[1.98804155574820$, 
TABLE 3: Comparison of modified-Gauss schemes for Orbit II.

\begin{tabular}{|c|c|c|c|c|c|c|c|}
\hline & $\left|\left(x_{1}-x_{0}\right)\right|$ & $\left|F\left(x_{1}\right)\right|$ & $\left|\left(x_{2}-x_{1}\right)\right|$ & $\left|F\left(x_{2}\right)\right|$ & $\left|\left(x_{3}-x_{2}\right)\right|$ & $\left|F\left(x_{3}\right)\right|$ & ACOC \\
\hline FP & $0.2397 e-1$ & - & $0.1132 e-2$ & - & $0.5163 e-4$ & - & 1.011 \\
\hline K8 & $0.2289 e-1$ & $0.2830 e-15$ & $0.2707 e-15$ & $0.7343 e-113$ & $0.7023 e-113$ & $0.5810 e-796$ & 7.007 \\
\hline S8 & $0.2289 e-1$ & $0.6075 e-17$ & $0.5809 e-17$ & $0.8328 e-142$ & $0.7964 e-142$ & $0.1039 e-1140$ & 8.006 \\
\hline M8 & $0.2289 e-1$ & $0.3696 e-17$ & $0.3534 e-17$ & $0.9933 e-144$ & $0.9500 e-144$ & $0.2705 e-1156$ & 8.005 \\
\hline T16 & $0.2289 e-1$ & $0.2913 e-45$ & $0.2786 e-45$ & $0.4103 e-748$ & $0.3924 e-748$ & 0.0 & 16.000 \\
\hline S16 & $0.2289 e-1$ & $0.1482 e-34$ & $0.1417 e-34$ & $0.4368 e-556$ & $0.4195 e-556$ & 0.0 & 16.010 \\
\hline M16 & $0.2289 e-1$ & $0.4590 e-34$ & $0.4389 e-34$ & $0.1062 e-557$ & $0.1016 e-557$ & 0.0 & 16.000 \\
\hline
\end{tabular}

TABLE 4: Comparison of modified-Gauss schemes for Orbit III.

\begin{tabular}{lccccccc}
\hline & $\left|\left(x_{1}-x_{0}\right)\right|$ & $\left|F\left(x_{1}\right)\right|$ & $\left|\left(x_{2}-x_{1}\right)\right|$ & $\left|F\left(x_{2}\right)\right|$ & $\left|\left(x_{3}-x_{2}\right)\right|$ & $\left|F\left(x_{3}\right)\right|$ & ACOC \\
\hline FP & $0.5499 e-1$ & - & $0.5830 e-2$ & - & $0.5723 e-3$ & 1.034 \\
K8 & $0.4968 e-1$ & $0.1579 e-11$ & $0.1437 e-11$ & $0.1661 e-85$ & $0.1512 e-85$ & $0.2376 e-603$ & 7.02 \\
S8 & $0.4968 e-1$ & $0.5842 e-13$ & $0.5317 e-13$ & $0.6265 e-109$ & $0.5701 e-109$ & $0.1095 e-876$ & 8.017 \\
M8 & $0.4968 e-1$ & $0.1092 e-13$ & $0.9941 e-14$ & $0.2294 e-115$ & $0.2087 e-115$ & $0.8667 e-929$ & 8.007 \\
T16 & $0.4968 e-1$ & $0.2742 e-34$ & $0.2495 e-34$ & $0.1560 e-567$ & $0.1419 e-567$ & $0.7 e-3998$ & 16.010 \\
S16 & $0.4968 e-1$ & $0.1550 e-26$ & $0.1411 e-26$ & $0.1066 e-435$ & $0.9702 e-436$ & $0.1 e-3998$ & 16.020 \\
M16 & $0.4968 e-1$ & $0.3967 e-27$ & $0.3610 e-27$ & $0.1512 e-445$ & $0.1376 e-445$ & $0.1 e-3998$ & 16.010 \\
\hline
\end{tabular}

measured in Earth radius (e.r.) on the Julian days (J.D.) from the perigee $t_{1}=0$ and $t_{2}=$ 0.01044412000000 . The orbital elements corresponding to the geometry of the orbit are the semimajor axis $a=4$ e.r., the eccentricity $e=0.2$, the epoch of the perigee $T_{0}=0 h 0 m 0 s$, and the Euler angles which fit the orbit in space are the right ascension of the ascending node, $\Omega=30^{\circ}$, the argument of the perigee $\omega=10^{\circ}$, and the inclination of the orbit $i=15^{\circ}$.

(ii) Test Orbit II has the following position vectors and times:

$\vec{r}_{1} \approx[-1.75981065999937$,

$1.68112802634201,1.16913429510899]$ e.r.,

$$
t_{1}=0 \text { J.D., }
$$

$\vec{r}_{2} \approx[-2.23077219993536$,

$0.77453561301361,1.34602197883025]$ e.r.,

$$
t_{2}=0.01527809 \text { J.D. }
$$

Orbital elements are $\Omega=80^{\circ}, \omega=60^{\circ}, i=30^{\circ}, a=$ 3 e.r., $e=0.1$, and $T_{0}=0 h 0 m 0 s$.

(iii) Test Orbit III has the following position vectors and times:

$$
\vec{r}_{1} \approx[0.41136206679761 \text {, }
$$$$
-1.66250000000000,0.82272413359522] \text { e.r., }
$$

$$
t_{1}=0 \text { J.D., }
$$

$\vec{r}_{2} \approx[0.97756752977209$,

$$
\begin{array}{r}
-1.64428006097667,-0.04236299091612] \text { e.r., } \\
t_{2}=0.01316924 \text { J.D. }
\end{array}
$$

Orbital elements are $\Omega=120^{\circ}, \omega=150^{\circ}, i=60^{\circ}$, $a=2$ e.r., $e=0.05$, and $T_{0}=0 h 0 m 0 s$.
We will compare the different error estimations at the first three iterations of the proposed eighth-order method M8 and the known schemes K8 and S8, and the sixteenthorder method M16 and the schemes T16 and S16. We also include, in Tables 2, 3, and 4, the approximated computational order of convergence (ACOC) (see [19]), in order to check the computational efficiency of the schemes related to their theoretical rate of convergence. This index is evaluated by the formula:

$$
p \approx A C O C=\frac{\log \left|\left(x_{k+1}-x_{k}\right) /\left(x_{k}-x_{k-1}\right)\right|}{\log \left|\left(x_{k}-x_{k-1}\right) /\left(x_{k-1}-x_{k-2}\right)\right|} .
$$

The different test orbits have been chosen with increasing angle $v_{2}-v_{1}$. It measures the spread in the observations and by the design of Gauss' procedure, it induces instability in the system when it gets higher. The difference between the true anomalies of the observations is, for the test orbits I to III, $12.23^{\circ}, 22.06^{\circ}$, and $31.46^{\circ}$, respectively. It can be observed in Tables 1-4 that, when the spread of the observations increases, the precision obtained in the calculations per step reduces at the same rate for any method of the same order.

It is clear that the application of high-order schemes to the problem of preliminary orbit calculation by Gauss procedure gets an important success, as the gain in speed and the precision obtained in the calculations are increased.

Let us note that the precision of the orbital elements calculated with the third estimation provided by any sixteenthorder method is total, as all the 4000 decimal digits of the solution considered as exact are reached with only three iterations.

\section{Conclusion}

We have extended the idea of other researchers for designing higher-order iterative methods by using weight function procedure. 
The Gaussian procedure for determining preliminary orbits has been modified in order to use modern and efficient iterative schemes of any optimal order of convergence and achieve high-level accuracy.

From the obtained results, it can be deduced that the proposed schemes are, at least, as competitive as recently published methods of the same order of convergence, being better in some cases. It has also shown to be robust enough to hold the theoretical order of convergence when an exigent precision is demanded.

\section{Conflict of Interests}

The authors declare that they have no conflict of interests regarding the publication of this paper.

\section{Acknowledgment}

This research was supported by Ministerio de Ciencia y Tecnología MTM2011-28636-C02-02 and FONDOCYT 20111-B1-33 República Dominicana.

\section{References}

[1] A. M. Ostrowski, Solution of Equations and System of Equations, Prentice-Hall, Englewood Cliffs, NJ, USA, 1964.

[2] H. T. Kung and J. F. Traub, "Optimal order of one-point and multipoint iteration," Journal of the Association for Computing Machinery, vol. 21, pp. 643-651, 1974.

[3] M. S. Petković, B. Neta, L. D. Vladimir Petković, and J. Džunić, Multipoint Methods for Solving Nonlinear Equations, Elsevier, New York, NY, USA, 2013.

[4] S. Artidiello, F. Chicharro, A. Cordero, and J. R. Torregrosa, "Local convergence and dynamical analysis of a new family of optimal fourth-order iterative methods," International Journal of Computer Mathematics, vol. 90, no. 10, pp. 2049-2060, 2013.

[5] C. Chun, M. Y. Lee, B. Neta, and J. Džunić, "On optimal fourthorder iterative methods free from second derivative and their dynamics," Applied Mathematics and Computation, vol. 218, no. 11, pp. 6427-6438, 2012.

[6] Y. I. Kim, "A triparametric family of three-step optimal eighthorder methods for solving nonlinear equations," International Journal of Computer Mathematics, vol. 89, no. 8, pp. 1051-1059, 2012.

[7] Y. Khan, M. Fardi, and K. Sayevand, "A new general eighthorder family of iterative methods for solving nonlinear equations," Applied Mathematics Letters, vol. 25, no. 12, pp. 22622266, 2012.

[8] J. Džunić and M. S. Petković, "A family of three-point methods of Ostrowski's type for solving nonlinear equations," Journal of Applied Mathematics, vol. 2012, Article ID 425867, 9 pages, 2012.

[9] F. Soleymani, M. Sharifi, and B. S. Mousavi, "An improvement of Ostrowski's and King's techniques with optimal convergence order eight," Journal of Optimization Theory and Applications, vol. 153, no. 1, pp. 225-236, 2012.

[10] R. Thukral, "New sixteenth-order derivative-free methods for solving nonlinear equations," The American Journal of Computational and Applied Mathematics, vol. 2, no. 3, pp. 112-118, 2012.

[11] J. R. Sharma, R. K. Guha, and P. Gupta, "Improved King's methods with optimal order of convergence based on rational approximations," Applied Mathematics Letters, vol. 26, no. 4, pp. 473-480, 2013.

[12] J. F. Traub, Iterative Methods for the Solution of Equations, Prentice-Hall, New York, NY, USA, 1964.

[13] C. Chun, "Some fourth-order iterative methods for solving nonlinear equations," Applied Mathematics and Computation, vol. 195, no. 2, pp. 454-459, 2008.

[14] R. F. King, "A family of fourth order methods for nonlinear equations," SIAM Journal on Numerical Analysis, vol. 10, pp. 876-879, 1973.

[15] L. Zhao, X. Wang, and W. Guo, "New families of eighthorder methods with high efficiency index for solving nonlinear equations," WSEAS Transactions on Mathematics, vol. 11, pp. 283-293, 2012.

[16] J. Džunić, M. S. Petković, and L. D. Petković, "A family of optimal three-point methods for solving nonlinear equations using two parametric functions," Applied Mathematics and Computation, vol. 217, no. 19, pp. 7612-7619, 2011.

[17] S. Weerakoon and T. G. I. Fernando, "A variant of Newton's method with accelerated third-order convergence," Applied Mathematics Letters, vol. 13, no. 8, pp. 87-93, 2000.

[18] P. R. Escobal, Methods of Orbit Determination, Robert E. Krieger Publishing Company, 1965.

[19] A. Cordero and J. R. Torregrosa, "Variants of Newton's method using fifth-order quadrature formulas," Applied Mathematics and Computation, vol. 190, no. 1, pp. 686-698, 2007. 


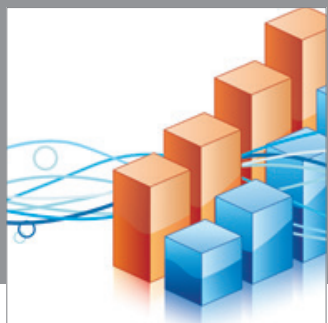

Advances in

Operations Research

mansans

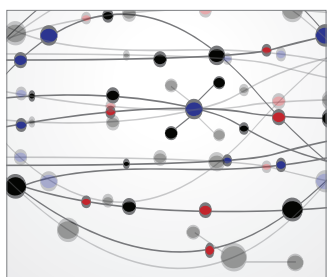

The Scientific World Journal
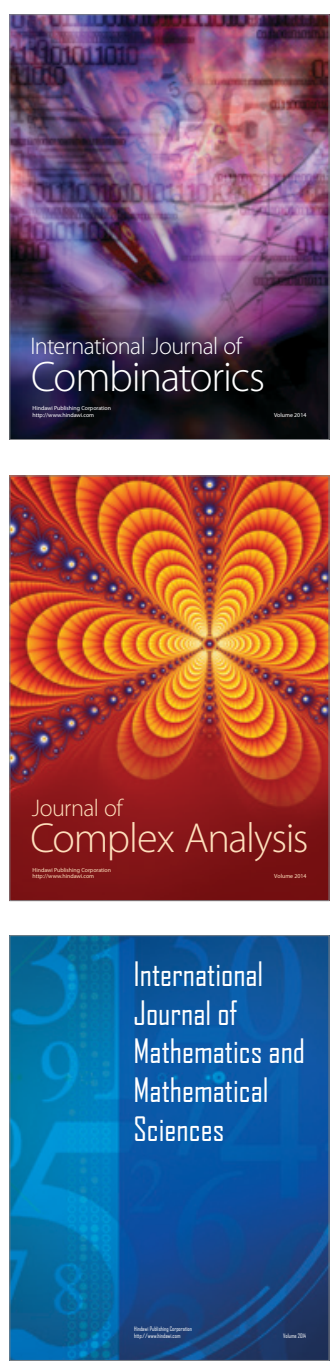
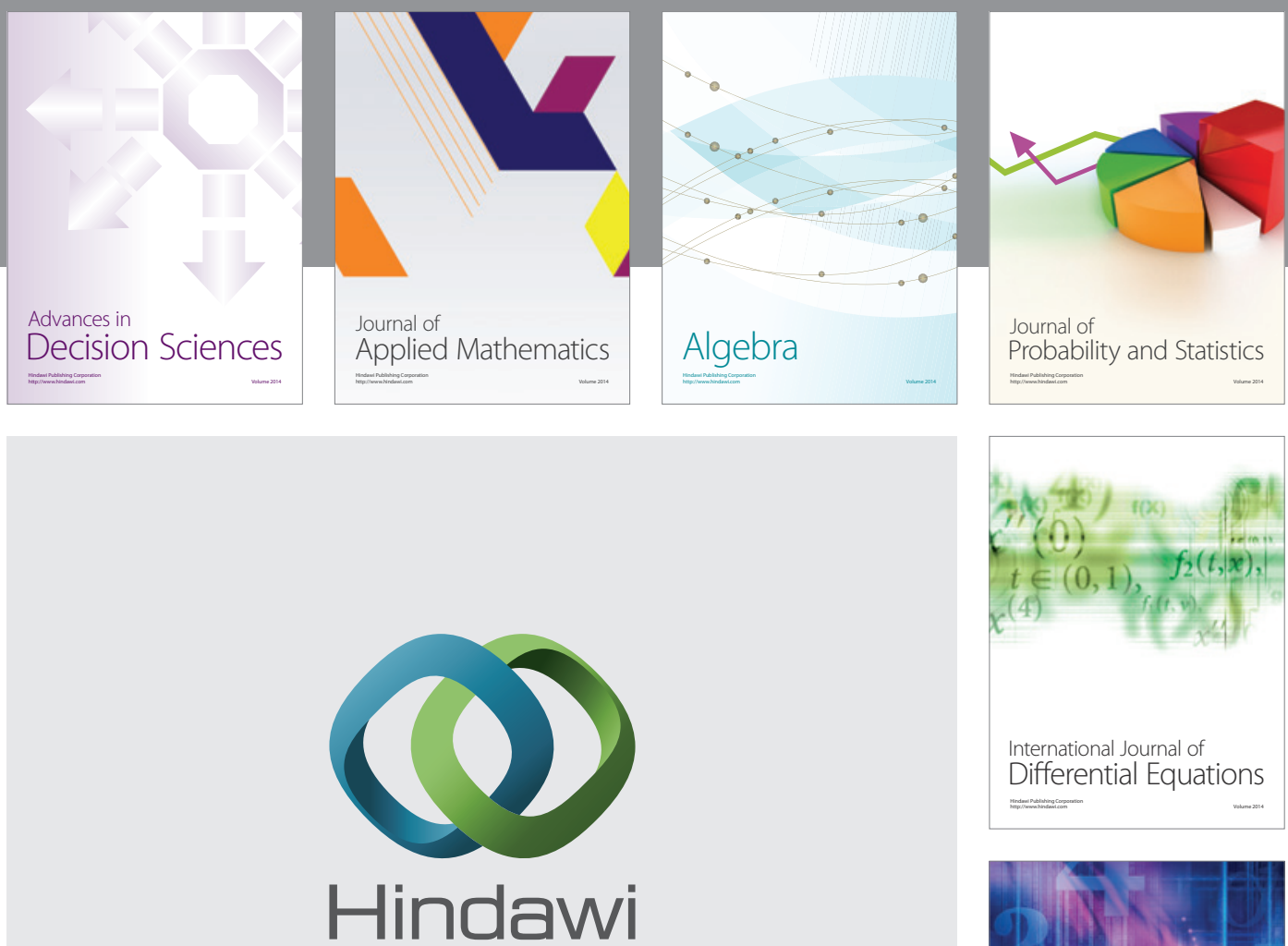

Submit your manuscripts at http://www.hindawi.com
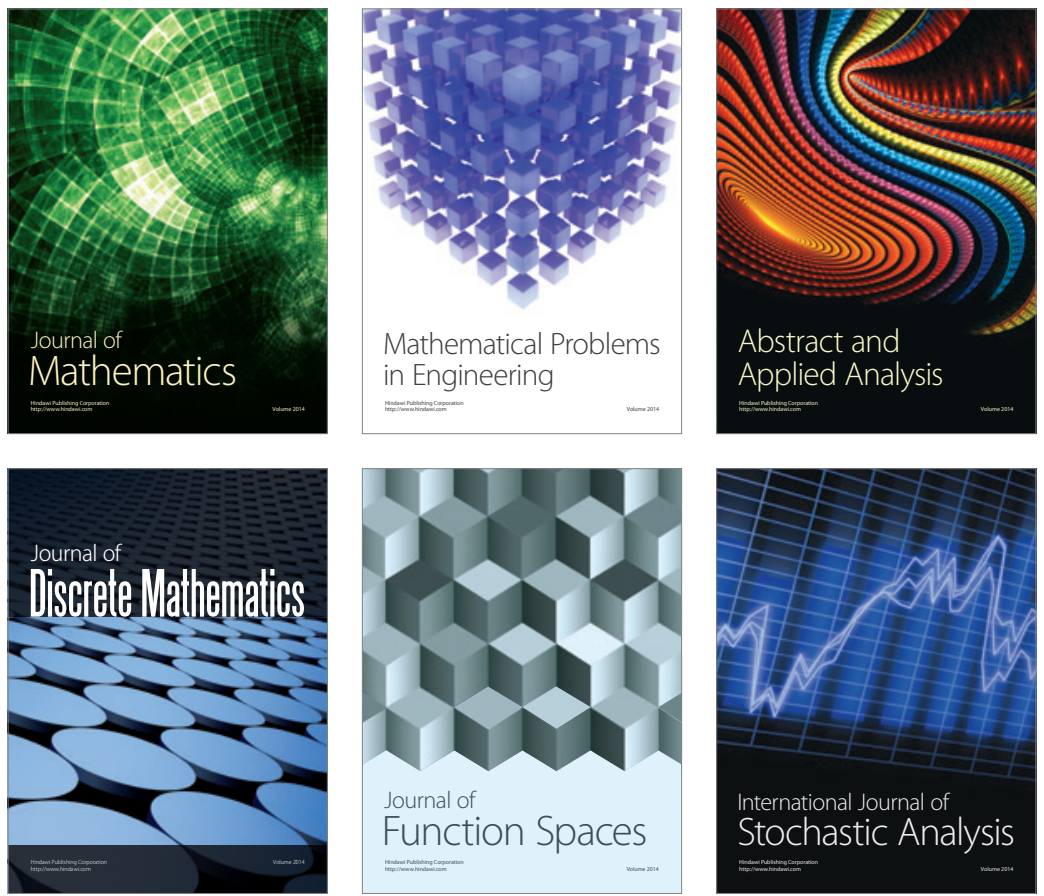

Journal of

Function Spaces

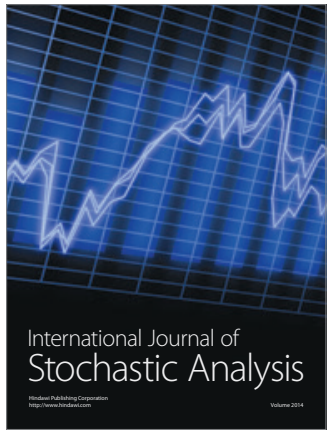

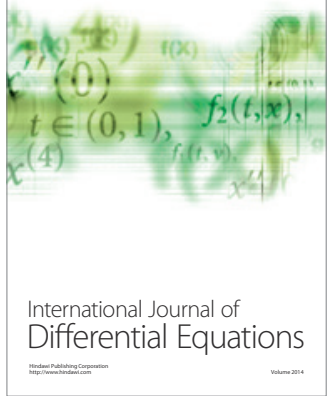
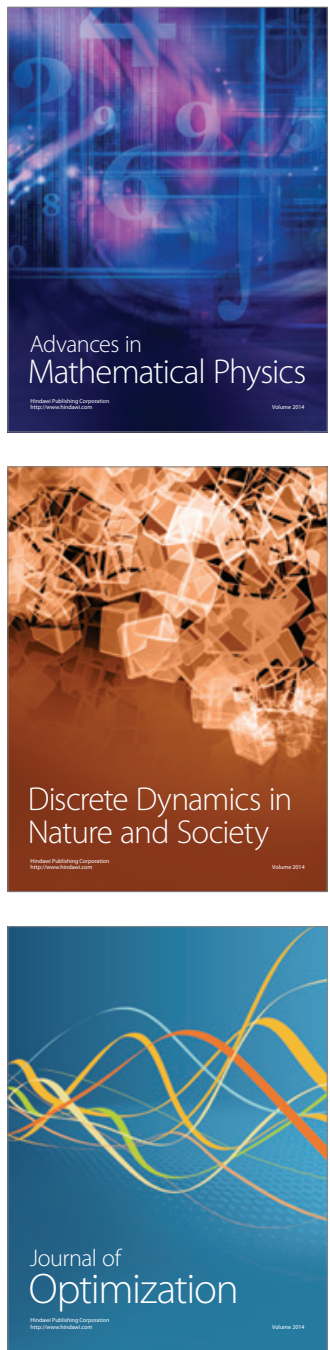